\title{
The Solar Orbiter Radio and Plasma Waves (RPW) instrument (Corrigendum)
}

\author{
M. Maksimovic ${ }^{1}{ }^{\circledR}$, S. D. Bale B $^{2,3,4}$, T. Chust $^{5}$, Y. Khotyaintsev ${ }^{12}$, V. Krasnoselskikh ${ }^{6}$, M. Kretzschmar ${ }^{6,7}$,
} D. Plettemeier ${ }^{8}$, H. O. Rucker ${ }^{35}$, J. Souček ${ }^{10}$, M. Steller ${ }^{9}, \breve{S}_{\text {. Štverák }}{ }^{11}$, P. Trávníček ${ }^{2,11}$, A. Vaivads ${ }^{12,21}$, S. Chaintreuil ${ }^{1}$, M. Dekkali ${ }^{1}$, O. Alexandrova ${ }^{1}$, P.-A. Astier ${ }^{1}$, G. Barbary ${ }^{1}$, D. Bérard ${ }^{1}$, X. Bonnin ${ }^{1}$, K. Boughedada ${ }^{1}$, B. Cecconi ${ }^{1}$, F. Chapron ${ }^{1}$, M. Chariet ${ }^{1,13}$, C. Collin ${ }^{1}$, Y. de Conchy ${ }^{1}$, D. Dias ${ }^{1}$, L. Guéguen ${ }^{1}$, L. Lamy ${ }^{1}$, V. Leray ${ }^{1,13}$, S. Lion ${ }^{1}$, L. R. Malac-Allain ${ }^{1}$, L. Matteini ${ }^{1}$, Q. N. Nguyen ${ }^{1}$, F. Pantellini ${ }^{1}$, J. Parisot ${ }^{1}$, P. Plasson $^{1}$, S. Thijs ${ }^{1}$, A. Vecchio ${ }^{1,34}$, I. Fratter ${ }^{14}$, E. Bellouard ${ }^{14}$, E. Lorfèvre ${ }^{14}$, P. Danto ${ }^{14}$, S. Julien ${ }^{15}$, E. Guilhem ${ }^{16}$, C. Fiachetti ${ }^{14}$, J. Sanisidro ${ }^{14}$, C. Laffaye ${ }^{14}$, F. Gonzalez ${ }^{14}$, B. Pontet ${ }^{14}$, N. Quéruel ${ }^{14}$, G. Jannet ${ }^{6}$, P. Fergeau ${ }^{6}$, J.-Y. Brochot ${ }^{6}$, G. Cassam-Chenai ${ }^{6}$,

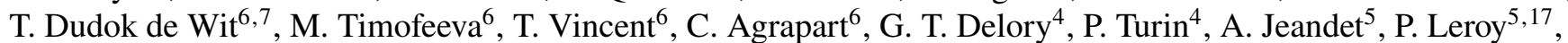
J.-C. Pellion ${ }^{5}$, V. Bouzid ${ }^{5}$, B. Katra ${ }^{5}$, R. Piberne ${ }^{5}$, W. Recart ${ }^{5,13}$, O. Santolík ${ }^{10,18}$, I. Kolmašováa ${ }^{10,18}$, V. Krupař ${ }^{10,19,20}$, O. Krupařová ${ }^{10}$, D. Píša ${ }^{10}$, L. Uhlîrir ${ }^{10}$, R. Lán ${ }^{10}$, J. Baše ${ }^{10}$, L. Ahlèn ${ }^{12}$, M. André ${ }^{12}$, L. Bylander ${ }^{21}$, V. Cripps ${ }^{12}$, C. Cully ${ }^{22}$, A. Eriksson ${ }^{12}$, S.-E. Jansson ${ }^{12}$, E. P. G. Johansson ${ }^{12}$, T. Karlsson ${ }^{21}$, W. Puccio ${ }^{12}$, J. Bř́nek ${ }^{11}$, H. Öttacher ${ }^{9}$, M. Panchenko ${ }^{9}$, M. Berthomier ${ }^{5}$, K. Goetz ${ }^{23}$, P. Hellinger ${ }^{11}$, T. S. Horbury ${ }^{24}$, K. Issautier ${ }^{1}$, E. Kontar ${ }^{25}$, S. Krucker ${ }^{26,2}$, O. Le Contel ${ }^{5}$, P. Louarn ${ }^{27}$, M. Martinović ${ }^{1,28,29}$, C. J. Owen ${ }^{30}$, A. Retino ${ }^{5}$, J. Rodríguez-Pacheco ${ }^{31}$, F. Sahraoui ${ }^{5}$, R. F. Wimmer-Schweingruber ${ }^{32}$, A. Zaslavsky ${ }^{1}$, and I. Zouganelis ${ }^{33}$

\footnotetext{
${ }^{1}$ LESIA, Observatoire de Paris, Université PSL, CNRS, Sorbonne Université, Univ. Paris Diderot, Sorbonne Paris Cité, 5 place Jules Janssen, 92195 Meudon, France

e-mail: milan.maksimovic@obspm. fr

${ }^{2}$ Space Sciences Laboratory, University of California, Berkeley, CA, USA

${ }^{3}$ Physics Department, University of California, Berkeley, CA, USA

${ }^{4}$ Stellar Scientific (now HELIOSPACE), 932 Parker St suite 2, Berkeley, CA 94710, USA

${ }^{5}$ LPP, CNRS, Ecole Polytechnique, Sorbonne Université, Observatoire de Paris, Université Paris-Saclay, PSL Research University, Palaiseau, Paris, France

${ }^{6}$ LPC2E, CNRS, 3A avenue de la Recherche Scientifique, Orléans, France

${ }^{7}$ Université d'Orléans, Orléans, France

8 Technische Universität Dresden, Würzburger Str. 35, 01187 Dresden, Germany

${ }^{9}$ Space Research Institute, Austrian Academy of Sciences, Graz, Austria

${ }^{10}$ Institute of Atmospheric Physics, Czech Academy of Sciences, Prague 14131, Czech Republic

${ }_{11}$ Astronomical Institute, Czech Academy of Sciences, 14100 Prague, Czech Republic

12 Swedish Institute of Space Physics (IRF), Uppsala, Sweden

${ }^{13}$ Nexeya Conseil et Formation, 5 Rue Boudeville ZI de Thibaud, 31100 Toulouse, France

${ }^{14}$ CNES, 18 Avenue Edouard Belin, 31400 Toulouse, France

${ }^{15}$ Logiqual, Avenue Didier Daurat, 31700 Blagnac, France

${ }_{16}$ Altran Sud Ouest 4, Avenue Didier Daurat, 31700 Blagnac, France

${ }^{17}$ Université de Rennes 1 - 263 Avenue du Général Leclerc, 35042 Rennes, France

${ }^{18}$ Faculty of Mathematics and Physics, Charles University, Prague, Czech Republic

${ }^{19}$ Universities Space Research Association, Columbia, MD, USA

${ }^{20}$ NASA Goddard Space Flight Center, Greenbelt, MD, USA

${ }^{21}$ Department of Space and Plasma Physics, School of Electrical Engineering and Computer Science, Royal Institute of Technology, Stockholm, Sweden

${ }^{22}$ Department of Physics and Astronomy, University of Calgary, Calgary, Alberta, Canada

${ }^{23}$ School of Physics and Astronomy, University of Minnesota, Minneapolis, MN, USA

${ }^{24}$ Department of Physics, Imperial College, SW7 2AZ London, UK

25 School of Astronomy and Astrophysics, Glasgow University, Glasgow, UK

${ }^{26}$ University of Applied Sciences and Arts Northwestern Switzerland, 5210 Windisch, Switzerland

27 IRAP, CNRS, Université de Toulouse, UPS, Toulouse, France

${ }^{28}$ Lunar and Planetary Laboratory, University of Arizona, Tucson, AZ 85719, USA

${ }^{29}$ Department of Astronomy, Faculty of Mathematics, University of Belgrade, Studentski trg 16, 11000 Belgrade, Serbia

${ }^{30}$ Mullard Space Science Laboratory, University College London, Holmbury St. Mary, Dorking, RH5 6NT, UK

${ }^{31}$ Space Research Group, Universidad de Alcala, Alcala de Henares, Spain

${ }^{32}$ Institute of Experimental and Applied Physics, Christian-Albrechts-University, Kiel, Germany

33 ESA, ESAC, Madrid, Spain

${ }^{34}$ Radboud Radio Lab, Department of Astrophysics/IMAPP - Radboud University, PO Box 9010, 6500GL Nijmegen, The Netherlands

${ }^{35}$ Commission for Astronomy, Austrian Academy of Sciences, Graz, Austria
}

A\&A, 642, A12 (2020), https://doi .org/10.1051/0004-6361/201936214

Key words. solar wind - instrumentation: miscellaneous - errata, addenda. 
The erratum concerns Fig. 9 entitled "Antenna radio-electrical properties" for which some of the parameters are not correct. The new figure with new parameters is provided in Fig. 1 of this corrigendum.

\begin{tabular}{|c|c|c|c|}
\hline & & Monopole & Dipole \\
\hline \multirow{3}{*}{$\begin{array}{c}\text { Antenna } \\
\text { capacitance } \\
C_{A}(p F)\end{array}$} & Physical length $L(m)$ & 6.5 & $\begin{array}{c}7.857 \text { for } V 1 V 2 \\
\text { and V1V3 } \\
6.99 \text { for } V 2 V 3\end{array}$ \\
\hline & radius $a(m)$ & 0.015 & 0.015 \\
\hline & $C_{A}(p F)$ for $f \ll c / 2 \pi L$ & 71.30 & $\begin{array}{c}41.54 \text { for V1V2 } \\
\text { and V1V3 } \\
37.80 \text { for V2V3 }\end{array}$ \\
\hline \multirow{5}{*}{ Cstray (pF) } & $\begin{array}{l}\text { antenna Cstray } \\
\text { ANT } 1 \text { / PZ } \\
\text { ANT } 2 \text { / PY } \\
\text { ANT } 3 \text { / MY }\end{array}$ & $\begin{array}{l}76.3 \pm 4.0 \\
78.9 \pm 3.0 \\
76.2 \pm 2.7\end{array}$ & \\
\hline & mean & $77.1 \pm 3.2$ & \\
\hline & preamplifier Cstray & 33.0 & \\
\hline & Stud Cstray & 0.0 & \\
\hline & Cstray & $109.5 \pm 3.2$ & $54.7 \pm 1.6$ \\
\hline$\Gamma=C_{A} /\left(C_{A}+C_{S}\right)$ & $\Gamma$ for $f \ll c / 2 \pi L$ & $0.394 \pm 0.007$ & $\begin{array}{c}0.431 \pm 0.007 \text { for } \\
\text { V1V2 and V1V3 } \\
0.408 \pm 0.007 \text { for } \\
\text { V2V3 }\end{array}$ \\
\hline \multirow{2}{*}{ Leff $(m)$} & $\min$ & 3.83 & 5.48 \\
\hline & $\max$ & 4.41 & 7.53 \\
\hline \multirow{2}{*}{$\begin{array}{c}\Gamma \text { Leff }(m) \text { for } \\
f \ll c / 2 \pi L\end{array}$} & $\min$ & 1.48 & 2.20 \\
\hline & $\max$ & 1.77 & 3.30 \\
\hline
\end{tabular}

Fig. 1. Corrected Antenna radio-electrical properties. 